Research Article

\title{
On Optimal M-Sets Related to Motzkin's Problem
}

\author{
Quan-Hui Yang $\mathbb{D}^{1},{ }^{1}$ Ting Pan $\mathbb{D}^{1},{ }^{1}$ and Jian-Dong $\mathrm{Wu} \mathbb{D}^{2}$ \\ ${ }^{1}$ School of Mathematics and Statistics, Nanjing University of Information Science and Technology, Nanjing 210044, China \\ ${ }^{2}$ School of Mathematical Sciences and Institute of Mathematics, Nanjing Normal University, Nanjing 210023, China
}

Correspondence should be addressed to Quan-Hui Yang; yangquanhui01@163.com

Received 6 May 2020; Revised 27 September 2020; Accepted 9 December 2020; Published 28 December 2020

Academic Editor: Li Guo

Copyright (c) 2020 Quan-Hui Yang et al. This is an open access article distributed under the Creative Commons Attribution License, which permits unrestricted use, distribution, and reproduction in any medium, provided the original work is properly cited.

Let $M$ be a set of positive integers. A set $S$ of nonnegative integers is called an $M$-set if $a$ and $b \in S$, then $a-b \notin M$. If $S \subseteq\{0,1, \ldots, n\}$ is an $M$-set with the maximal cardinality, then $S$ is called a maximal $M$-set of $\{0,1, \ldots, n\}$. If $S \cap\{0,1, \ldots, n\}$ is a maximal $M$-set of $\{0,1, \ldots, n\}$ for all integers $n \geq 0$, then we call $S$ an optimal $M$-set. In this paper, we study the existence of an optimal $M$-set.

\section{Introduction}

Let $\mathbb{N}$ be the set of all nonnegative integers. For a positive real number $x$ and $S \subseteq \mathbb{N}$, we denote by $S(x)$ the number of elements $n \in S$ such that $n \leq x$. The upper and lower densities of $S$ denoted by $\bar{\delta}(S)$ and $\underline{\delta}(S)$, respectively, are given by

$$
\begin{aligned}
& \bar{\delta}(S):=\lim _{x \longrightarrow \infty} \sup \frac{S(x)}{x}, \\
& \underline{\delta}(S):=\lim _{x \longrightarrow \infty} \inf \frac{S(x)}{x} .
\end{aligned}
$$

If $\bar{\delta}(S)=\underline{\delta}(S)$, let $\delta(S)=\bar{\delta}(S)=\underline{\delta}(S)$, then we say that $S$ has density $\delta(S)$.

Given a set $M$ of positive integers, we call $S \subseteq \mathbb{N}$ an $M$-set if $a \in S$ and $b \in S$, then $a-b \notin M$. Motzkin [1] presented the problem of determining the quantity

$$
\mu(M):=\sup _{S} \bar{\delta}(S)
$$

where the supremum is taken over all $M$-sets $S$. Cantor and Gordon [2] proved that if $|M|=1, \mu(M)=(1 / 2)$ and that if $M=\left\{m_{1}, m_{2}\right\}$, then $\mu(M)=\left(\left\lfloor\left(m_{1}+m_{2}\right) / 2\right\rfloor /\left(m_{1}+m_{2}\right)\right)$. Later, Haralambis [3] determined $\mu(M)$ for many members of the families $\{1, j, k\}$ and $\{1,2, j, k\}$. In 1999, Gupta and Tripathi [4] completely determined $\mu(M)$ when $M$ is a finite arithmetic progression. In 2011, Pandey and Tripathi [5] investigated this quantity when $M$ is related to an arithmetic progression. After that, Pandey and Tripathi [6] and the first author and Tang [7] determined $\mu(M)$ when $M$ is a geometric progression. For other results, one may refer to [8-11].

In 2010, Chen and Yang [12] and Khovanova and Konyagin [13] studied the upper density among sets of nonnegative integers in which no two elements have quotient belonging to $M$.

Suppose $G$ is a graph and $T$ is a set of nonnegative integers. A $T$-coloring of $G$ is an assignment of a positive integer $f(x)$ to each vertex $x$ of $G$ so that if $x$ and $y$ are joined by an edge of $G$, then $f(x)-f(y)$ is not in $T$. $T$-colorings were introduced by Hale [14] in connection with the channel assignment problem in communications. Motzkin's problem has connections with the $T$-colouring problem. For more results on this topic, one can refer to [15-19].

Motzkin's problem also has connections with some other problems, such as problems related to the fractional chromatic number of distance graphs and the Lonely Runner Conjecture. One can refer to [9, 20-29].

In this paper, for any integers $a$ and $b$, we define $[a, b]=\{a \leq n \leq b: n \in \mathbb{Z}\}$. For an $M$-set $S \subseteq[0, n]$, if $|S| \geq\left|S^{\prime}\right|$ for any $M$-set $S^{\prime} \subseteq[0, n]$, then $S$ is said to be a 
maximal $M$-set of $[0, n]$. If $S \cap[0, n]$ is a maximal $M$-set of $[0, n]$ for all integers $n \geq 0$, then we call $S$ an optimal $M$-set. If $M$ is finite, then an optimal $M$-set is infinite. For a finite set $M$, let $G(M)$ be the infinite sequence $0, a_{1}, a_{2}, \ldots$ recursively by choosing $a_{k+1}$ to be the least integer exceeding $a_{k}$ such that $\left\{0, a_{1}, a_{2}, \ldots, a_{k+1}\right\}$ is also an $M$-set. It is easy to see that, for a given set $M$, if there exists an optimal $M$-set, then this set must be $G(M)$.

In this paper, we determine all the optimal $M$-set when $|M| \leq 2$.

Theorem 1. For any set $M$ with $|M|=1$, there exists an optimal $M-$ set.

Theorem 2. Let $M=\left\{m_{1}, m_{2}\right\}$, where $m_{1}<m_{2}$, and let $\operatorname{gcd}\left(m_{1}, m_{2}\right)=d$. Then, there exists an optimal $M-$ set if and only if $m_{1} \mid m_{2}$ or $m_{2}=k m_{1} \pm d$ with an odd $k$.

Remark 1. By Theorem 2, we know that there exists an optimal $M$-set when $m_{1}=2$.

\section{Proofs of Theorem 1}

Before presenting the proof of Theorem 1, we first give some lemmas here. Lemma 1 is a small generalization of Corollary 1 , which is used by Cantor and Gordon in [2].

Lemma 1. Let $M_{1}=\left\{m_{1}, m_{2}, \ldots\right\}$ and $M_{2}=\left\{d m_{1}\right.$, $\left.d m_{2}, \ldots\right\}$, where $d$ is a positive integer. For any $M_{1}-$ sets $S_{0}, S_{1}, \ldots, S_{d-1}$,

$$
S=\bigcup_{i=0}^{d-1}\left\{d a+i: a \in S_{i}\right\}
$$

is an $M_{2}$-set. Conversely, for any $M_{2}$-set $T$ and $i \in\{0,1, \ldots, d-1\}$,

$$
T_{i}=\left\{\frac{a-i}{d}: a \in T, a \equiv i(\bmod d)\right\}
$$

is an $M_{1}-$ set.

Proof. Suppose that there exist two elements $u, v \in S$ such that $u-v=d m_{i}$ for some positive integer $i$. Let $u=d a+i$ and $v=$ $d b+j$ with $a, b \in S_{j}$ and $0 \leq i, j \leq d-1$. Then, by $d \mid u-v$, we obtain $i=j$. Hence, $a, b \in S_{i}$ and $a-b=m_{i} \in M_{1}$. This contradicts that $S_{i}$ is an $M_{1}$-set.

Conversely, suppose that there exists an integer $i$ with $0 \leq i \leq d-1$ such that $T_{i}$ is not an $M_{1}-$ set. Then, there exist $x, y \in T_{i}$ such that $x-y=m_{i}$ for some positive integer $i$. Let $x=((m-i) / d), y=((n-i) / d)$, where $m$ and $n \in T$. Then, $m-n=d m_{i} \in M_{2}$. This contradicts that $T$ is an $M_{2}-$ set.

Corollary 1. Let $M_{1}=\left\{m_{1}, m_{2}, \ldots\right\}$ and $M_{2}=\left\{d m_{1}\right.$, $\left.d m_{2}, \ldots\right\}$, where $d$ is a positive integer. For any $M_{1}-$ sets $S$, the set $\cup_{i=0}^{d-1}\{d a+i: a \in S\}$ is an $M_{2}-$ set.

Corollary 1 follows from Lemma 1 immediately by taking $S_{0}=S_{1}=\cdots=S_{d-1}=S$.

For two sets $A$ and $B$ and an integer $d$, let $A+B=\{a+b: a \in A, b \in B\}$,
$A-B=\{a-b: a \in A, b \in B\}, d \cdot A=\{d a: a \in A\}$, and $d+$ $A=\{d+a: a \in A\}$.

Lemma 2. Let $M_{1}=\left\{m_{1}, m_{2}, \ldots\right\}$ and $M_{2}=\left\{d m_{1}, d m_{2}, \ldots\right\}$, where $d$ is a positive integer. Then, $G\left(M_{1}\right)$ is an optimal $M_{1}-$ set if and only if $G\left(M_{2}\right)$ is an optimal $M_{2}-$ set.

Proof. We first prove the relationship between $G\left(M_{1}\right)$ and $G\left(M_{2}\right)$, i.e.,

$$
G\left(M_{2}\right)=d \cdot G\left(M_{1}\right)+D, \quad \text { where, } D=\{0,1, \ldots, d-1\} \text {. }
$$

Suppose that equality (5) does not hold. Let $n$ be the smallest integer such that $G\left(M_{2}\right) \cap[0, n] \neq\left(d \cdot G\left(M_{1}\right)+\right.$ $D) \cap[0, n]$. For any integer $m \leq n$, let $m=q_{m} d+r_{m}$, where $0 \leq r_{m} \leq d-1$. Then, $n \in G\left(M_{2}\right)$ is equivalent to $n-m \notin M_{2}$ for all $m<n$ and $m \in G\left(M_{2}\right)$. If $r_{m} \neq r_{n}$, then $n-m \notin M_{2}$ must hold. Hence, we only need to consider the case $r_{m}=r_{n}$. So, $n \in G\left(M_{2}\right)$ is equivalent to $q_{n}-q_{m} \notin M_{1}$ for all integers $q_{m}$ with $q_{m}<q_{n}$ and $q_{m} \in G\left(M_{1}\right)$, that is, $q_{n} \in G\left(M_{1}\right)$. Hence, $n \in G\left(M_{2}\right)$ is equivalent to $n \in d \cdot G\left(M_{1}\right)+D$, a contradiction.

Now, we shall prove $G\left(M_{1}\right)$ is an optimal $M_{1}-$ set if and only if $G\left(M_{2}\right)$ is an optimal $M_{2}$-set.

Suppose that $G\left(M_{2}\right)$ is an optimal $M_{2}-$ set. If $G\left(M_{1}\right)$ is not an optimal $M_{1}-$ set, then there exists an integer $n_{1}$ and an $M_{1}$-set $S_{1} \subseteq\left[0, n_{1}\right]$ such that $\left|S_{1}\right|>\left|G\left(M_{1}\right) \cap\left[0, n_{1}\right]\right|$. Let $S_{2}=d \cdot S_{1}+D$. Then, $S_{2} \subseteq\left[0, d n_{1}+d-1\right]$. By (5), we have $\left|S_{2}\right|>\left|G\left(M_{2}\right) \cap\left[0, d n_{1}+d-1\right]\right|$. By Lemma $1, S_{2}$ is an $M_{2}$-set. Thus, $G\left(M_{2}\right)$ is not an optimal $M_{2}$-set.

Now, suppose that $G\left(M_{1}\right)$ is an optimal $M_{1}-$ set. If $G\left(M_{2}\right)$ is not an optimal $M_{2}-$ set, then there exists an integer $n_{2}$ and an $M_{2}$-set $S_{2} \subseteq\left[0, n_{2}\right]$ such that $\left|S_{2}\right|>\left|G\left(M_{2}\right) \cap\left[0, n_{2}\right]\right|$.

Suppose that $n_{2} \in G\left(M_{2}\right)$. Let $n_{2}^{\prime}$ be the maximal integer such that $n_{2}^{\prime} \leq n_{2}$ and $n_{2}^{\prime} \equiv d-1(\bmod d)$. Then, by $(5)$, we have $\left[n_{2}^{\prime}+1, n_{2}\right] \subseteq G\left(M_{2}\right)$. Thus,

$$
\left|S_{2} \cap\left[0, n_{2}^{\prime}\right]\right|>\left|G\left(M_{2}\right) \cap\left[0, n_{2}^{\prime}\right]\right| .
$$

Suppose that $n_{2} \notin G\left(M_{2}\right)$. Let $n_{2}^{\prime \prime}$ be the minimal integer such that $n_{2}^{\prime \prime} \geq n_{2}$ and $n_{2}^{\prime \prime} \equiv d-1(\bmod d)$. Then, by (5), we have $\left[n_{2}, n_{2}^{\prime \prime}\right] \cap G\left(M_{2}\right)=\phi$. Thus,

$$
\left|S_{2} \cap\left[0, n_{2}^{\prime \prime}\right]\right|>\left|G\left(M_{2}\right) \cap\left[0, n_{2}^{\prime \prime}\right]\right| .
$$

Hence, without loss of generality, we may assume $n_{2} \equiv d-1(\bmod d)$. By $(5)$, we have

$$
G\left(M_{2}\right) \cap\left[0, n_{2}\right]=d \cdot\left(G\left(M_{1}\right) \cap\left[0, \frac{\left(n_{2}-d+1\right)}{d}\right]\right)+D \text {. }
$$

For $\quad 0 \leq i \leq d-1, \quad$ let $\quad T_{i}=\left\{((a-i) / d): a \in S_{2}\right.$, $a \equiv i(\bmod d)\} . \quad$ Then, $T_{i} \subseteq\left[0,\left(\left(n_{2}-d+1\right) / d\right)\right]$. Since $\left|S_{2}\right|>\left|G\left(M_{2}\right) \cap\left[0, n_{2}\right]\right|$, by $(8)$, there exists an integer $i_{0}$ with $0 \leq i_{0} \leq d-1$ such that $\left|T_{i_{0}}\right|>\left|G\left(M_{1}\right) \cap\left[0,\left(\left(n_{2}-d+1\right) / d\right)\right]\right|$. By Lemma 1, $T_{i_{0}}$ is an $M_{1}$-set. Thus, $G\left(M_{1}\right)$ is not an optimal $M_{1}$-set. 
Therefore, $G\left(M_{1}\right)$ is an optimal $M_{1}-$ set if and only if $G\left(M_{2}\right)$ is an optimal $M_{2}$-set.

Proof of Theorem 1: it suffices to prove that $G(M)$ is an optimal $M$-set when $|M|=1$. By Lemma 2, we may assume that $M=\{1\}$. Clearly, $G(M)$ is the set of all even numbers. Since any $M$-set $S$ contains no consecutive integers, it follows that $G(M)$ is an optimal $M$-set.

\section{Proofs of Theorem 2}

In order to prove Theorem 2, we first prepare some lemmas. Let $|x|_{m}$ denote the absolute value of the absolutely least remainder of $x(\bmod m)$. The proof of Lemma 3 follows proofs of Theorem 1 and Theorem 4 (ii) in [2]. For the convenience of the reader, we present it here.

Lemma 3 (see [2], Theorem 1 and Theorem 4 (ii)). Let $M=\left\{m_{1}, m_{2}\right\}$, where $\operatorname{gcd}\left(m_{1}+m_{2}\right)=1$ and $2 \mid m_{1}+m_{2}$. Then, there exists an $M-$ set $S \subseteq\left[0, m_{1}+m_{2}-1\right]$ such that $|S|=\left(\left(m_{1}+m_{2}-1\right) / 2\right)$.

Proof. By $\operatorname{gcd}\left(m_{1}+m_{2}\right)=1$ and $2 \mid m_{1}+m_{2}$, we have $\operatorname{gcd}\left(m_{2}-m_{1}, m_{1}+m_{2}\right)=1$, and then there exists $c$ such that $c\left(m_{2}-m_{1}\right) \equiv 1\left(\bmod m_{1}+m_{2}\right)$. Thus, $\quad c m_{2}-c m_{1} \equiv$ $1\left(\bmod m_{1}+m_{2}\right) . \quad$ Noting that $\quad c m_{2}+c m_{1} \equiv 0$ $\left(\bmod m_{1}+m_{2}\right)$, we obtain $2 c m_{2} \equiv 1\left(\bmod m_{1}+m_{2}\right)$ and $2 c m_{1} \equiv-1\left(\bmod m_{1}+m_{2}\right)$. Hence, $2 c m_{1} \equiv-2 c m_{2} \equiv-1$ $\equiv m_{1}+m_{2}-1\left(\bmod m_{1}+m_{2}\right)$, and then,

$$
c m_{1} \equiv-c m_{2} \equiv \frac{m_{1}+m_{2}-1}{2}\left(\bmod m_{1}+m_{2}\right) .
$$

Let

$$
\begin{aligned}
S & =\bigcup_{r=0}^{\left(m_{1}+m_{2}-1\right) / 2-1}\left\{n \in \mathbb{Z}: 0 \leq n \leq m_{1}+m_{2}-1, c n\right. \\
& \left.\equiv r\left(\bmod m_{1}+m_{2}\right)\right\} .
\end{aligned}
$$

Then, $S \subseteq\left[0, m_{1}+m_{2}-1\right]$ and $|S|=\left(m_{1}+m_{2}-1\right) / 2$. Next, we shall prove that $S$ is an $M$-set. That is, if $a$ and $b \in S$, then $a-b \notin M$. We obtain $c a=q_{1}\left(m_{1}+m_{2}\right)+$ $r_{1}$ and $c b=q_{2}\left(m_{1}+m_{2}\right)+r_{2}$, where $0 \leq r_{1}, r_{2}<\left(m_{1}+\right.$ $\left.m_{2}-1\right) / 2$. Then, if $a-b \in M$, we have

$$
c(a-b)=\left(q_{1}-q_{2}\right)\left(m_{1}+m_{2}\right)+\left(r_{1}-r_{2}\right)=c m_{1} \text { or } c m_{2} \text {. }
$$

It follows that $r_{1}-r_{2} \equiv c m_{1}$ or $c m_{2}\left(\bmod m_{1}+m_{2}\right)$. However, by $0 \leq r_{1}, r_{2}<\left(m_{1}+m_{2}-1 / 2\right)$ and (9), we have

$$
\left|r_{1}-r_{2}\right|<\frac{m_{1}+m_{2}-1}{2}=\left|c m_{1}\right|_{m_{1}+m_{2}}=\left|c m_{2}\right|_{m_{1}+m_{2}},
$$

a contradiction.

In the next lemma, we study the period of $G(M)$.

Lemma 4. Let $M=\left\{m_{1}, m_{2}\right\}$. Then, $G(M)$ is periodic with period $m_{1}+m_{2}$.

Proof. We shall prove that $n \in G(M)$ if and only if $n+$ $m_{1}+m_{2} \in G(M)$ for all $n \geq 0$.
If $n \in G(M)$, then $n+m_{1}$ and $n+m_{2} \notin G(M)$. By the definition of $G(M)$, we have $n+m_{1}+m_{2} \in G(M)$.

If $n \notin G(M)$, then we shall prove $n+m_{1}+m_{2} \notin G(M)$. Suppose that $n+m_{1}+m_{2} \in G(M)$. Then, $n+m_{1}$ and $n+$ $m_{2} \notin G(M)$. If $n+m_{2}-m_{1} \notin G(M)$, by $n \notin G(M)$ and the definition of $G(M)$, we have $n+m_{2} \in G(M)$, a contradiction. Thus, we have $n+m_{2}-m_{1} \in G(M)$, and then $n-m_{1} \notin G(M)$. Similarly, we also have $n+m_{1}$ $m_{2} \in G(M)$ and $n-m_{2} \notin G(M)$. Since $n-m_{1} \notin G(M)$ and $n-m_{2} \notin G(M)$, by the definition of $G(M)$, it follows that $n \in G(M)$, a contradiction. Hence, $n+m_{1}+m_{2} \notin G(M)$.

Therefore, $G(M)$ is a periodic set with period $m_{1}+m_{2}$.

In 2012, the authors of [30] study the difference sets containing integer powers. Lemma 5 is the main theorem in [30], which is used to prove Lemma 6.

Lemma 5 (see [30], Theorem 1). Let $n>m \geq 2$ be positive integers and $n=(m+1) l+r$, where $0 \leq r \leq m$. Let

$$
\tau(m, n)= \begin{cases}\left\lfloor\frac{n}{2}\right\rfloor+1, & \text { if } m \text { is odd, } \\ \left(\frac{m l}{2}\right)+\delta, & \text { if } m \text { is even, }\end{cases}
$$

where $\delta$ denotes the cardinality of even numbers in the interval $[0, \min \{r, m-2\}]$. Then, we have the following:

(i) If $C$ is a subset of $[0, n]$ with $|C|>\tau(m, n)$, then $C-C$ contains either 1 or $m$

(ii) There exists a subset $C$ of $[0, n]$ with $|C|=\tau(m, n)$ such that $C-C$ contains no power of $m$

Lemma 6. Let $M=\left\{1, m_{2}\right\}$. Then, $G(M)$ is an optimal $M-s e t$.

Proof. It is easy to see that, if $m_{2}$ is odd, then $G(M)$ is the set of all even integers; if $m_{2}$ is even, then

$$
G(M)=\bigcup_{k=0}^{\infty} A_{k}, \quad \text { where } A_{k}=k\left(m_{2}+1\right)+2 \cdot\left[0, \frac{m_{2}}{2}-1\right] .
$$

Hence, $|G(M) \cap[0, n]|=\tau\left(m_{2}, n\right)$. By Lemma $5(\mathrm{i})$, there is no set $C \subseteq[0, n]$ such that $|C|>|G(M) \cap[0, n]|$ and $C$ is also an $M$-set. Hence, $G(M)$ is an optimal $M$-set.

Proof of Theorem 2: it suffices for us to determine $M$ such that $G(M)$ is an optimal $M$-set. By Lemma 2, we may assume $d=1$. If $m_{1}=1$, by Lemma 6 , then $G(M)$ is an optimal $M$-set. Now, we suppose that $m_{1}>1$. Noting that $\left(m_{1}, m_{2}\right)=1$, we write $m_{2}=k m_{1}+r$, where $1 \leq r \leq m_{1}-1$. If $k$ is odd, then

$$
G(M) \cap\left[0, m_{1}+m_{2}-1\right]=\underset{j=0}{((k-1) / 2)}\left\{2 j m_{1}+s: 0 \leq s \leq m_{1}-1\right\},
$$

and so $\left|G(M) \cap\left[0, m_{1}+m_{2}-1\right]\right|=\left((k+1) m_{1} / 2\right)$. If $k$ is even, then 


$$
\begin{aligned}
G(M) \cap\left[0, m_{1}+m_{2}-1\right] & =\bigcup_{j=0}^{(k / 2)-1}\left\{2 j m_{1}+s: 0 \leq s \leq m_{1}-1\right\} \\
& \cup\left[k m_{1}, k m_{1}+r-1\right] .
\end{aligned}
$$

It follows that $\left|G(M) \cap\left[0, m_{1}+m_{2}-1\right]\right|=$ $\left(k m_{1} / 2\right)+r$.

Case 1. $2\left|m_{1}, 2\right| m_{2}$. Let $M^{\prime}$ be the set of all even numbers contained in $\left[0, m_{1}+m_{2}-1\right]$. Clearly, $M^{\prime}$ is an $M-$ set and $\left|M^{\prime}\right|=\left(\left(m_{1}+m_{2}\right) / 2\right)$. Since $\left(\left(m_{1}+m_{2}\right) / 2\right)>\left((k+1) m_{1} / 2\right)$ and $\left(\left(m_{1}+m_{2}\right) / 2\right)>\left(k m_{1} / 2\right)+r$, we have $\left|M^{\prime}\right|>\mid G(M) \cap$ $\left[0, m_{1}+m_{2}-1\right] \mid$. Hence, $G(M)$ is not an optimal $M-$ set.

Case 2. $2 \mid m_{1}+m_{2}$. By Lemma 3, there exists an $M$-set $S \subseteq\left[0, m_{1}+m_{2}-1\right]$ such that $|S|=\left(\left(m_{1}+m_{2}-1\right) / 2\right)$. It is easy to check that $|S|>\left|G(M) \cap\left[0, m_{1}+m_{2}-1\right]\right|$ except for the following two cases: (a) $k$ is odd and $r=1$ and (b) $k$ is even and $r=m_{1}-1$. Hence, $G(M)$ is not an optimal $M$-set except for these two cases.

Now, we shall prove that $G(M)$ is an optimal $M$-set in cases (a) and (b). By Lemma $4, G(M)$ is periodic with period $m_{1}+m_{2}$. Hence, it suffices for us to prove that $G(M) \cap[0, n]$ is a maximal $M-$ set of $[0, n]$ for any integer $n \in\left[0, m_{1}+m_{2}-1\right]$.

Subcase 1: $k$ is odd and $r=1$. Then, $m_{2}=k m_{1}+1$. Suppose that $T \subseteq\left[0, m_{1}+m_{2}-1\right]$ is an $M$-set. Noting that $m_{1} \in M$, we have

$$
\left|T \cap\left\{2 j m_{1}+i,(2 j+1) m_{1}+i\right\}\right| \leq 1,
$$

for all integers $i, j$ with $0 \leq i \leq m_{1}-1$ and $0 \leq j \leq$ $((k-1) / 2)$. Since

$$
\left\{2 j m_{1}+i: 0 \leq i \leq m_{1}-1,0 \leq j \leq \frac{(k-1)}{2}\right\} \subseteq G(M) .
$$

It follows that $G(M) \cap[0, n]$ is a maximal $M$-set of $[0, n]$ for all $n \leq(k+1) m_{1}-1$.

Now, we consider the case $n=(k+1) m_{1}=$ $m_{1}+m_{2}-1$. If $|T|>\left|G(M) \cap\left[0, m_{1}+m_{2}-1\right]\right|$, then we have $(k+1) m_{1} \in T$ and

$$
\left|T \cap\left\{2 j m_{1}+i,(2 j+1) m_{1}+i\right\}\right|=1,
$$

for all integers $i, j$ with $0 \leq i \leq m_{1}-1$ and $0 \leq j \leq((k-1) / 2)$. Hence,

$$
\begin{aligned}
(k+1) m_{1} \in T & \stackrel{m_{2} \in M}{\Rightarrow} m_{1}-1 \notin T \stackrel{(3.2)}{\Rightarrow} 2 m_{1}-1 \in T \\
& \stackrel{(3.2)}{\Rightarrow} \cdots \stackrel{(3.2)}{\Rightarrow}(k+1) m_{1}-1 \in T \\
& \stackrel{m_{2} \in M}{\Rightarrow} m_{1}-2 \notin T \stackrel{(3.2)}{\Rightarrow} 2 m_{1}-2 \in T \\
& \stackrel{(3.2)}{\Rightarrow} \cdots \stackrel{(3.2)}{\Rightarrow}(k+1) m_{1}-2 \in T .
\end{aligned}
$$

Similarly, we have $\left\{(k+1) m_{1}-3,(k+1) m_{1}-4, \ldots\right.$, $\left.k m_{1}\right\} \subseteq T$. On the other hand, by $(k+1) m_{1} \in T$ and $m_{1} \in M$, we have $k m_{1} \notin T$, a contradiction. Thus, $G(M)$ is an optimal $M$-set in this case.

Subcase 2: $k$ is even and $r=m_{1}-1$. Then, $m_{2}=(k+1) m_{1}-1$. Suppose that $T^{\prime} \subseteq\left[0, m_{1}+m_{2}-1\right]$ is an $M$-set. By $m_{1} \in M$, we have

$$
\left|T^{\prime} \cap\left\{2 j m_{1}+i,(2 j+1) m_{1}+i\right\}\right| \leq 1,
$$

for all integers $i$, jwith $0 \leq i \leq m_{1}-1$ and $0 \leq j \leq(k / 2)-1$. However,

$$
\bigcup_{j=0}^{k / 2-1}\left\{2 j m_{1}+s: 0 \leq s \leq m_{1}-1\right\} \cup\left[k m_{1}, m_{2}-1\right] \subseteq G(M) .
$$

It follows that $G(M) \cap[0, n]$ is a maximal $M$-set of $[0, n]$ for all $n \leq m_{2}-1$.

Now, we consider the case $m_{2} \leq n \leq m_{1}+m_{2}-1$. If $G(M) \cap\left[0, m_{1}+m_{2}-1\right]$ is a maximal $M$-set of $\left[0, m_{1}+m_{2}-1\right]$, by $\left[n+1, m_{1}+m_{2}-1\right] \cap G(M)=\phi$, then $G(M) \cap[0, n]$ is a maximal $M$-set of $[0, n]$. Hence, it is enough for us to prove $G(M) \cap\left[0, m_{1}+m_{2}-1\right]$ is a maximal $M$-set of $\left[0, m_{1}+m_{2}-1\right]$. If $\left|T^{\prime}\right|>\mid G(M) \cap$ $\left[0, m_{1}+m_{2}-1\right] \mid$, then we have

$$
\left|T^{\prime} \cap\left\{2 j m_{1}+i,(2 j+1) m_{1}+i\right\}\right|=1,
$$

for all integers $i, j$ with $0 \leq i \leq m_{1}-1$ and $0 \leq j \leq(k / 2)$. In particular, $(k+1) m_{1}-1 \in T^{\prime}$, since $(k+2) m_{1}-1=$ $m_{1}+m_{2} \notin T^{\prime}$. Hence,

$$
\begin{aligned}
(k+1) m_{1}-1 \in T^{\prime} & \stackrel{m_{2} \in M}{\Rightarrow} 0 \notin T^{\prime} \stackrel{(3.3)}{\Rightarrow} m_{1} \in T^{\prime} \stackrel{(3.3)}{\Rightarrow} \ldots \\
& \stackrel{(3.3)}{\Rightarrow} k m_{1} \notin T^{\prime} \stackrel{(3.3)}{\Rightarrow}(k+1) m_{1} \in T^{\prime} \\
& \stackrel{m_{2} \in M}{\Rightarrow} 1 \notin T^{\prime} \stackrel{(3.3)}{\Rightarrow} \ldots \stackrel{(3.3)}{\Rightarrow} k m_{1}+1 \notin T^{\prime} .
\end{aligned}
$$

Similarly, we obtain $\left[k m_{1}+2,(k+1) m_{1}-1\right] \cap T^{\prime}=\phi$. It is a contradiction with $(k+1) m_{1}-1 \in T^{\prime}$. Hence, $G(M)$ is also an optimal $M$-set in this case.

Therefore, $G(M)$ is an optimal $M$-set if and only if $m_{1}=$ 1 or $m_{2}=k m_{1} \pm 1$, where $k$ is odd.

At the end of this paper, we pose a problem for further research.

Problem 1. Given an integer $k \geq 3$, determine all sets $M$ with $|M|=k$ such that there exists an optimal $M$-set.

Remark 2. If $G(M)$ is optimal, then $G(M)$ gives the value of $\mu(M)$. If $G(M)$ is not optimal, we cannot find any information about the value of $\mu(M)$. Thus, Problem 1 and Motzkin's problem are incomparable.

\section{Data Availability}

No data were used to support this study. 


\section{Conflicts of Interest}

The authors declare that they have no conflicts of interest.

\section{Acknowledgments}

This study was supported by the National Natural Science Foundation for Youth of China (Grant no. 11501299), Natural Science Foundation of Jiangsu Province (Grant nos. BK20150889 and 15KJB110014), and the Startup Foundation for Introducing Talent of NUIST (Grant no. 2014r029).

\section{References}

[1] T. S. Motzkin: Unpublished problem collection.

[2] D. G. Cantor and B. Gordon, "Sequences of integers with missing differences," Journal of Combinatorial Theory, Series A, vol. 14, no. 3, pp. 281-287, 1973.

[3] N. M. Haralambis, "Sets of integers with missing differences," Journal of Combinatorial Theory, Series A, vol. 23, no. 1, pp. 22-33, 1977.

[4] S. Gupta and A. Tripathi, "Density of $M$-sets in arithmetic progression," Acta Arithmetica, vol. 89, no. 3, pp. 255-257, 1999.

[5] R. K. Pandey and A. Tripathi, "On the density of integral sets with missing differences from sets related to arithmetic progressions," Journal of Number Theory, vol. 131, no. 4, pp. 634-647, 2011.

[6] R. K. Pandey and A. Tripathi, "A note on the density of $M$-sets in geometric sequence," Ars Combinatoria-Waterloo then Winnipeg, vol. 119, pp. 221-224, 2015.

[7] Q. H. Yang and M. Tang, "Density of integral sets with missing differences," Ars Combinatoria-Waterloo then Winnipeg, vol. 132, pp. 231-239, 2017.

[8] S. Gupta, "Sets of integers with missing differences," Journal of Combinatorial Theory, Series A, vol. 89, no. 1, pp. 55-69, 2000.

[9] D. D.-F. Liu and G. Robinson, "Sequences of integers with three missing separations," European Journal of Combinatorics, vol. 85, 2020.

[10] R. K. Pandey, Some results on the density of integral sets with missing differences, Ph.D thesis, Department of Mathematics, Indian Institute of Technology, Delhi, India, 2008.

[11] R. K. Pandey and A. Tripathi, "A note on a problem of motzkin regarding density of integral sets with missing differences," Journal of Integer Sequences, vol. 14, no. 6, 2011.

[12] Y.-G. Chen and H.-X. Yang, "Sequences of integers with missing quotients," Discrete Mathematics, vol. 310, no. 5, pp. 1105-1111, 2010.

[13] T. Khovanova and S. Konyagin, "Sequences of integers with missing quotients and dense points without neighbors," Discrete Mathematics, vol. 312, no. 10, pp. 1776-1787, 2012.

[14] W. K. Hale, "Frequency assignment: theory and applications," Proceedings of the IEEE, vol. 68, no. 12, pp. 1497-1514, 1980.

[15] D. D.-F. Liu, Graph homomorphisms and the channel assignment problem, Ph.D. thesis, University of South Carolina, Columbia, SC, USA, 1991.

[16] D. D.-F. Liu, "T-colorings of graphs," Discrete Mathematics, vol. 101, no. 1-3, pp. 203-212, 1992.

[17] D. D.-F. Liu, "On a conjecture of T-colorings," in Proceedings of the Twenty-Fifth Southeastern International Conference on Combinatorics, Graph Theory and Computing, vol. 103, pp. 27-31, Boca Raton, FL, USA, March 1994.

[18] D. D.-F. Liu, "T-graphs and the channel assignment problem," Discrete Mathematics, vol. 161, no. 1-3, pp. 197-205, 1996.
[19] D. D.-F. Liu, "T-colorings and chromatic number of distance graphs," Ars Combinatoria-Waterloo then Winnipeg, vol. 56, pp. 65-80, 2000.

[20] W. Bienia, L. Goddyn, P. Gvozdjak, A. Sebő, and M. Tarsi, "Flows, view obstructions, and the lonely runner," Journal of Combinatorial Theory, Series B, vol. 72, no. 1, pp. 1-9, 1998.

[21] J. Barajas and O. Serra, "The lonely runner with seven runners," Electronic Journal of Combinatorics, vol. 15, p. 18, 2008.

[22] J. M. Carraher, D. Galvin, S. G. Hartke, A. J. Radcliffe, and D. Stolee, "On the independence ratio of distance graphs," Discrete Mathematics, vol. 339, no. 12, pp. 3058-3072, 2016.

[23] G. J. Chang, L. Huang, and X. Zhu, "Circular chromatic numbers and fractional chromatic numbers of distance graphs," European Journal of Combinatorics, vol. 19, no. 4, pp. 423-431, 1998.

[24] G. J. Chang, D. D.-F. Liu, and X. Zhu, "Distance graphs and T-coloring," Journal of Combinatorial Theory, Series B, vol. 75, no. 2, pp. 259-269, 1999.

[25] D. D.-F. Liu, "From rainbow to the lonely runner: a survey on coloring parameters of distance graphs," Taiwanese Journal of Mathematics, vol. 12, no. 4, pp. 851-871, 2008.

[26] D. D.-F. Liu and X. Zhu, "Distance graphs with missing multiples in the distance sets," Journal of Graph Theory, vol. 30, no. 4, pp. 245-259, 1999.

[27] D. D.-F. Liu and X. D. Zhu, "Fractional chromatic number and circular chromatic number for distance graphs with large clique size," Journal of Graph Theory, vol. 47, Article ID 129146, 2004.

[28] D. D.-F. Liu and X. Zhu, "Fractional chromatic number of distance graphs generated by two-interval sets," European Journal of Combinatorics, vol. 29, no. 7, pp. 1733-1743, 2008.

[29] J. Z. Wu and W. S. Lin, "Circular chromatic numbers and fractional chromatic numbers of distance graphs with distance sets missing an interval," Ars Combinatoria-Waterloo then Winnipeg, vol. 70, Article ID 161168, 2004.

[30] Q.-H. Yang and J.-D. Wu, "Sum and difference sets containing integer powers," Czechoslovak Mathematical Journal, vol. 62, no. 3, pp. 787-793, 2012. 